\title{
Activation of S1PR2 upregulates tumoral DPD by promoting the interaction of TWIST1 with the JMJD3-RNA Pol II complex bound to the DPYD promoter
}

\author{
Zhi-Kun Guo ${ }^{1}$, Ying-Zhi Zhang1, Yu-Hang Zhang ${ }^{2}$, Dong-Dong Luo ${ }^{3}$, Sheng-Biao Wan ${ }^{3}$, \\ Xianjun $\mathrm{Qu}^{1}$, and shuxiang cui ${ }^{1}$ \\ ${ }^{1}$ Capital Medical University \\ ${ }^{2}$ Peking University First Hospital \\ ${ }^{3}$ Ocean University of China School of Medicine and Pharmacy
}

February 1, 2022

\begin{abstract}
Background and Purpose: Dihydropyrimidine dehydrogenase (DPD) is a major determinant of 5-FU resistance in cancers. DPD catalyzes 5-FU into FBAL ( $\alpha$-fluoro- $\beta$-alanine) to lower intracellular 5 -FU level. We aimed to investigate mechanism and clinical significance of FBAL-stimulated sphingosine 1-phosphate receptor 2 (S1PR2) in upregulation of DPD in colonic cancer. Experimental Approach: Cancer cells transfected or silenced S1PR2 were exposed to FBAL for analyzed S1PR2 and DPD levels. Luciferase reporter assay analyzed S1PR2-activated TWIST1 binding to DPYD promoter. Co-IP assay analyzed TWIST1 interaction with JMJD3-RNA Pol II complex binding to DPYD promoter. HT-29sh-S1PR2 or SW480TgS1PR2 cells xenografted nude mice were used to evaluate clinical significance of S1PR2-upregulated tumoral DPD. Key Results: Activation of S1PR2 upregulated DPD in colonic cancer cells and human fresh cancer specimens. FBAL was first time identified as an etiological stimulator of S1PR2 activation. The FBAL-stimulated S1PR2 increased TWIST1 binding to DPYD promoter and interacting with JMJD3-RNA Pol II complex, enhancing H3K27me3-enriched DPYD transcription elongation. Transfection of S1PR2 in SW480TgS1PR2 xenograft contributed 5-FU resistance by 45.14\%, and silence of S1PR2 improved 5-FU sensitivity by $62.12 \%$ in HT-29sh-S1PR2 xenograft. S1PR2 inhibitor JTE013 prevented the FBAL-stimulated S1PR2's effects in upregulating DPD. Cancer cells with high S1PR2 are more resistant to 5-FU, strongly suggesting clinical significance that combination use of S1PR2 inhibitors may be the appropriate 5-FU-based regimens of colonic cancers. Conclusions and Implications: The FBALstimulated S1PR2 increased TWIST1 binding to DPYD promoter and interacting with JMJD3-RNA Pol II, enhancing the H3K27me3-enriched DPYD transcription elongation.
\end{abstract}

\section{Hosted file}

manuscript.doc available at https://authorea.com/users/458424/articles/554988-activation-ofs1pr2-upregulates-tumoral-dpd-by-promoting-the-interaction-of-twist1-with-the-jmjd3-rnapol-ii-complex-bound-to-the-dpyd-promoter 